\title{
Differences in food intake and diet quality in vegans, vegetarians and omnivores in Belgium
}

\author{
Amy Mullee ${ }^{1,2}$, Laura Fiers ${ }^{3}$, Willem De Keyzer ${ }_{5}^{3}$, Tom Deliens ${ }^{4}$, Peter Clarys ${ }^{4}$, Patrick Mullie ${ }^{4}$, \\ Peter Deriemaeker ${ }^{4}$, Barbara Vanaelst ${ }^{5}$, Rachel Wasson ${ }^{2}$, Tobias Leenaert ${ }^{6}$, \\ Stefaan De Henauw, Marc J. Gunter ${ }^{2}$ and Inge Huybrechts ${ }^{2,4}$ \\ ${ }^{1}$ School of Public Health, Physiotherapy and Population Science, University College Dublin (UCD), Dublin, Republic \\ of Ireland, ${ }^{2}$ International Agency for Research on Cancer (IARC), Lyon, France, ${ }^{3}$ University College Ghent, \\ Department of Bio- and Food Sciences, Ghent, Belgium, ${ }^{4}$ Faculty of Physical Education and Physiotherapy, \\ Department Movement and Sport Sciences, Vrije Universiteit Brussel, Brussels, Belgium, ${ }^{5}$ Ghent University, \\ Department of Public Health, UZ-Ghent, Ghent, Belgium and ${ }^{6}$ EVA vzw, Ghent, Belgium
}

Meat, meat products and other animal-derived foods are major sources of certain vitamins, minerals and amino-acids ${ }^{(1)}$. Consequently, there is a risk that when animal products are insufficiently substituted by nutritious plant-based foods, a vegetarian or vegan diet could possibly lead to inadequacies for certain nutrients. Little is known about the quality of the diet of Belgian vegans and (semi-)vegetarians in comparison with Belgian omnivores and their compliance with the food based dietary guidelines (FBDG) ${ }^{(2)}$.

The aim of this cross-sectional study was to examine the diet quality of vegans, vegetarians, semi-vegetarians and omnivores using a diet quality index (DQI) ${ }^{(3)}$ especially developed alongside the Belgian food based dietary guidelines (FBDG) and compared these dietary patterns in a representative sample of the Belgian population.

A representative sample $(n=3,039)$ from the 2004 Belgian food consumption survey ${ }^{(4)}$ and additional participants recruited through a Belgian vegetarian organization and Universities in Ghent $(n=1,803)$ completed an online questionnaire including a selfreported food frequency questionnaire (FFQ). Totaling $n=123$ vegans, $n=623$ vegetarians, $n=704$ semi-vegetarians and $n=3,392$ omnivores, of which $72 \%$ were female. A DQI based on Belgian FBDG and consisting of the four index parameters: meal, diversity, equilibrium and quality was calculated and compared between dietary patterns.

None of the dietary pattern groups were fully compliant with the FBDG. However, all dietary pattern groups did meet the minimum recommendation for protein sources. Recommendations for dairy or calcium-fortified drinks were not met in any of the dietary pattern groups, although vegans, vegetarians, and semi-vegetarians consumed at least 1.7 times more milk or soya drinks than omnivores. DQI scores were significantly higher in vegan men and vegetarian and semi-vegetarian women compared to omnivores $(\mathrm{P}<0 \cdot 05)$.

In conclusion, vegans, vegetarians and semi-vegetarians appear to adequately compensate for the absence or reduced intake of animal protein, and conform to the FBDG as much as, or better than omnivores. However, none of the dietary patterns complied with all the Belgian dietary recommendations.

The work that Amy Mullee contributed to in this abstract was undertaken during the tenure of an IARC-Ireland Postdoctoral Fellowship from the International Agency for Research on Cancer, funded by the Irish Cancer Society.

1. Phillips F (2005) Vegetarian Nutrition. London: British Nutrition Foundation.

2. Vigez (2012) The Active Food Guide Pyramid. Belgium: Het Vlaams Instituut voor Gezondheidspromotie en Ziektepreventie (VIGeZ).

3. Huybrechts I, Vereecken C, De Bacquer D et al. (2010) Br J Nutr 104, 135-44.

4. De Vriese S, Huybrechts I, Moreau M et al. (2006) The Belgian Food Consumption Survey 1-2004: Report (Enquête de consommation alimentaire Belge 1-2004: Rapport). Report No.: D/2006/2505/16. Brussel: Afdeling Epidemiologie. 